\title{
Plastic Flow in a Thin Layer: the Theory, Formulations of the Boundary- Value Problems and the Applications
}

\author{
Vagid Kadymov ${ }^{1}$, Elena Yanovskaya ${ }^{2}$ \\ ${ }^{1}$ Technological University- MIREA, RU-119484, Moscow, Russia \\ ${ }^{2}$ Moscow State Technological University "STANKIN", RU-127055, Moscow, Russia
}

\begin{abstract}
Two-dimensional, averaged over the thickness of the layer, mathematical theory of the spreading of a plastic layer on the plane has been studied. General and simplified mathematical formulations of boundary value problem were presented. The problem of plastic stretching of a strip by forces applied on its "clamped" ends was investigated. The analysis of various modes of the process was carried out, which are determined by both the total compression force of the ends and the total tensile force. Mathematical analogy between the process of the free spreading of a plastic layer on the plane and the process of heat transfer was studied. For known forms of a domain occupied by a thin plastic layer at the initial time and for a given law of convergence of the plates, the evolution of the boundary of a plastic layer spreading was described. The exact particular solutions of the aforementioned problem was obtained.
\end{abstract}

\section{Introduction}

Problems of plastic flow of the material in a relatively thin layer between the converging surfaces of the tool bodies form a fairly wide class. This class includes most of the technological problems of material treatment by pressure, such as stamping and pressing of thin-walled structural elements, thin-sheet rolling and many others. These are complex space problems of plastic shaping with a variety of parameters that determine the properties of the material. In these processes high pressures develop, which are an order of magnitude higher than the shear properties of the layer material, so that in the initial approximation, the properties of the material in the layer are close to those of a hydrodynamic liquid [1-3]. Almost along the entire contact surface, slippage is observed, in which the contact surfaces coincide with the sliding surfaces and the friction forces on them reach a maximum value equal to the yield strength of the layer material [2]. The accuracy requirements of the final part define another feature of these processes. As known [2], high contact pressures cause normal elastic movements of the working surfaces of the tool bodies, commensurate with the thickness of the plastic layer. Therefore, tin this case, ignoring elastic movements of the tool bodies becomes unjustified. In high-speed processes of material treatment by pressure the viscosity and inertia forces play an essential role, which greatly complicate modeling of the physical processes. In these processes, temperature and heat flows play an important role. Thus, heat generation due to the sliding friction forces on the contact surface and the dissipation of mechanical energy should also be considered.
Based on the analysis of the solution of L.Prandtl's problem, A.A. Ilyushin has formulated a number of hypotheses regarding the kinematics and the friction forces on the contact. He then utilized them to develop an effective two-dimensional theory of flow in a thin plastic layer that allowed to efficiently explore these processes. Within the framework of this model, a general boundary value problem for a "viscous" liquid has been formulated. This problem is described by nonlinear partial differential equations of the second order with respect to three unknown functions: contact pressure and two components of the flow velocity [1, 2, 5, 6]. It should be noted that until recently, all studies in this area were conducted on the basis of a partial simplified formulation of the boundary value problem in the model of an "ideal" fluid described by nonlinear partial differential equations of the first order, in which tangent stresses are neglected. In the framework of the simplified formulation, the kinematics of the spreading of the plastic layer was investigated and the evolutionary equation determining the free boundary of the spreading layer was obtained. In [3] other forms of representation of the evolutionary equation have been received. What's more, it has been established that this equation is reduced to a special case of the nonlinear equation of thermal conductivity.

This theory has been extended to the case of plastic flow on elastically deformable surfaces [2] and on surfaces with a pronounced texture (anisotropy of the properties of friction forces on contact). The theory has also been applied for the flows in high-speed processes and in the treatment processes of material volumetrically compressible and material with structural dependence (powder, sintered material). 


\section{Equations and mathematics}

General statement of the boundary-value problem of the spreading of a plastic layer. The basic equations of the boundary-value problem of plastic layer flow on the plane have the form $[1,2,5]$ :

$$
\begin{gathered}
\frac{\partial p}{\partial x}=\frac{\sigma_{s}}{3} \frac{d t}{d \lambda} \Delta u-\frac{\tau_{s}}{h}\left(\frac{u-u_{1}}{\sqrt{\left(u-u_{1}\right)^{2}+(v-v)^{2}}}\right) \\
\left.+\frac{u-u_{2}}{\sqrt{\left(u-u_{1}\right)^{2}+(v-v)^{2}}}\right), \\
\frac{\partial p}{\partial y}=\frac{\sigma_{s}}{3} \frac{d t}{d \lambda} \Delta v-\frac{\tau_{s}}{h}\left(\frac{v-v_{1}}{\sqrt{\left(u-u_{1}\right)^{2}+(v-v)^{2}}}\right), \\
\left.+\frac{v-v_{2}}{\sqrt{\left(u-u_{1}\right)^{2}+(v-v)^{2}}}\right) \\
\frac{\partial u}{\partial x}+\frac{\partial v}{\partial y}-\frac{d \lambda}{d t}=0,
\end{gathered}
$$

where $p, u, v$ are the contact pressure and components of flow velocity; $\vec{v}_{1}=\left(u_{1}, v_{1}\right)$ and $\vec{v}_{2}=\left(u_{2}, v_{2}\right)$ - the known speeds of internal movements of surfaces of tool bodies relative to a fixed geometric surface; $\sigma_{S}=\sqrt{3} \tau_{S}$ is the yield strength of plastic material; $h=h(t)$ is the known law of change of the layer thickness; $\lambda(t)=$ $\ln \left(h\left(t_{0}\right) / h(t)\right)$ is the degree of deformation, where $t_{0}$ is some initial point of time. In particular, if there are no internal motions of physical surfaces $\left(\vec{v}_{1}=\vec{v}_{2}=0\right)$, then the differential equations of quasi -static equilibrium are simplified:

$$
\begin{aligned}
& \frac{\partial \mathrm{p}}{\partial \mathrm{x}}=\frac{\sigma_{\mathrm{s}}}{3} \frac{\mathrm{dt}}{\mathrm{d} \lambda} \Delta \mathrm{u}-\frac{2 \tau_{\mathrm{s}}}{\mathrm{h}} \frac{\mathrm{u}}{\sqrt{\mathrm{u}^{2}+\mathrm{v}^{2}}} \\
& \frac{\partial \mathrm{p}}{\partial \mathrm{y}}=\frac{\sigma_{\mathrm{s}}}{3} \frac{\mathrm{dt}}{\mathrm{d} \lambda} \Delta \mathrm{v}-\frac{2 \tau_{\mathrm{s}}}{\mathrm{h}} \frac{\mathrm{v}}{\sqrt{\mathrm{u}^{2}+\mathrm{v}^{2}}}
\end{aligned}
$$

On a free previously unknown boundary the two scalar dynamic conditions are specified:

$\vec{\sigma}^{(n)}=\tilde{\sigma} \cdot \vec{n}=\left(\sigma_{x x} n_{x}+\sigma_{x y} n_{y} ; \sigma_{x y} n_{x}+\sigma_{y y} n_{y}\right)=\overrightarrow{0}$

and the kinematic condition is given:

$$
v_{n} \equiv \vec{v} \cdot \vec{n}=-\frac{\frac{\partial F}{\partial t}}{|\operatorname{grad} F|}
$$

In (2.4)” $\vec{n}=\left(n_{x}, n_{y}\right)=\nabla F /|\nabla F|$ is the normal vector to the boundary, and the components of the stress tensor $\tilde{\sigma}$ have the form:

$$
\begin{gathered}
\sigma_{x x}=-p+\frac{2}{3} \sigma_{s}+\frac{2}{3} \sigma_{s} \frac{d t}{d \lambda} \frac{\partial u}{\partial x} \\
\sigma_{x y}=\frac{1}{3} \sigma_{s} \frac{d t}{d \lambda}\left(\frac{\partial u}{\partial y}+\frac{\partial v}{\partial x}\right) \\
\sigma_{y y}=-p+\frac{2}{3} \sigma_{s}+\frac{2}{3} \sigma_{s} \frac{d t}{d \lambda} \frac{\partial v}{\partial y}
\end{gathered}
$$

At the tool contact boundary (usually known) $\Gamma_{\sigma}: F(x, y, t)=0$, the following two conditions are set: one dynamic condition with respect to the shear stress

$$
\begin{gathered}
\left|\vec{\sigma}^{(n)} \cdot \vec{\tau}\right|=\mid \sigma_{x x} n_{x} n_{y}+\sigma_{[y}\left(n_{x}^{2}+n_{y}^{2}\right)- \\
\sigma_{y y} n_{x} n_{y} \mid=\mu \tau_{s}, 0 \leq \mu \leq 1
\end{gathered}
$$

and one kinematic condition of the impermeability

$$
v_{n} \equiv \vec{v} \cdot \vec{n}=-\frac{\partial G / \partial t}{|\operatorname{grad} G|}
$$

where $\vec{\tau}$ and $\vec{n}$ are the unitary tangent and normal vectors with respect to $\Gamma_{c}$. In particular, at the fixed boundary, the normal velocity is zero.

Let's move on to dimensionless variables. Let $\sigma_{s}, L_{0}, h_{0},\left(d h\left(t_{0}\right) / d t\right)$ be the characteristic values of the yield strength of the material, the linear size and thickness of the layer, and the rate of convergence of the plates. We introduce a small parameter $\varepsilon=h_{0} / L_{0}<<1$, characterizing the fineness of the plastic layer. The analysis of incompressibility equation leads to: a) $t_{0}=L_{0} / v_{0}$; b) $v_{0}=-\left(d h\left(t_{0}\right) / d t\right) / \varepsilon$. The latter means that the characteristic value of the flow velocity along the layer is much greater than the rate of convergence of the plates, which is commonly expected. Let's consider $p_{0}$ as the characteristic value of the contact pressure in the plastic layer. The analysis of the quasistatic equilibrium equations shows that the characteristic value of the contact pressure is much higher than the yield strength of the layer material, i.e. $p_{0}=\sigma_{s} / \varepsilon$. We now have the following system of equations in dimensionless form:

$$
\begin{gathered}
\frac{\partial \bar{p}}{\partial \bar{x}}=\varepsilon \frac{d \bar{t}}{d \lambda} \bar{\Delta} \bar{u}-\frac{2}{\sqrt{3}} \frac{1}{\bar{h}} \frac{\bar{u}}{\sqrt{\bar{u}^{2}+\bar{v}^{2}}}, \\
\frac{\partial \bar{p}}{\partial \bar{y}}=\varepsilon \frac{d \bar{t}}{d \lambda} \bar{\Delta} \bar{v}-\frac{2}{\sqrt{3}} \frac{1}{\bar{h}} \frac{\bar{v}}{\sqrt{\bar{u}^{2}+\bar{v}^{2}}},
\end{gathered}
$$




$$
\frac{\partial \bar{u}}{\partial \bar{x}}+\frac{\partial \bar{v}}{\partial \bar{y}}-\frac{d \lambda}{d \bar{t}}=0
$$

where dimensionless quantities are marked by a dash at the top, $\bar{h}=h / h\left(t_{0}\right)$.

The conditions at the free boundary $\Gamma_{\sigma}: \bar{F}(\bar{x}, \bar{y}, \bar{t})=0$ :

$\left[-\overline{\mathrm{p}}+\left(\frac{2}{3}+\frac{2}{3} \frac{\overline{\mathrm{dt}} \mathrm{d} \lambda}{\partial \mathrm{u}}\right) \varepsilon\right] \mathrm{n}_{\mathrm{x}}+\left[\frac{1}{3} \frac{\overline{\mathrm{dt}}}{\mathrm{d} \lambda}\left(\frac{\overline{\mathrm{u}}}{\partial \mathrm{y}}+\frac{\overline{\partial \mathrm{v}}}{\partial \mathrm{x}}\right) \varepsilon\right] \mathrm{n}_{\mathrm{y}}=0$,

$$
\begin{gathered}
{\left[\frac{1}{3} \frac{\overline{\mathrm{dt}}}{\mathrm{d} \lambda}\left(\frac{\overline{\partial \mathrm{u}}}{\partial \mathrm{y}}+\frac{\overline{\partial \mathrm{v}}}{\partial \mathrm{x}}\right) \varepsilon\right] \mathrm{n}_{\mathrm{x}}+\left[-\overline{\mathrm{p}}+\left(\frac{2}{3}+\frac{2}{3} \frac{\overline{\mathrm{dt}}}{\mathrm{d} \lambda} \frac{\overline{\partial v}}{\partial \mathrm{y}}\right) \varepsilon\right] \mathrm{n}_{\mathrm{y}}} \\
=0,
\end{gathered}
$$

$$
\bar{v}_{n}=-\frac{\partial \bar{F} / \partial \bar{t}}{|\operatorname{grad} \bar{F}|}
$$

The conditions at the tool contact boundary $\Gamma_{C}$ :

$$
\begin{gathered}
{\left[-\bar{p}+\left(\frac{2}{3}+\frac{2}{3} \frac{\overline{d t}}{d \lambda} \frac{\overline{\partial u}}{\partial x}\right) \varepsilon\right] n_{x} n_{y}+\left[\frac{1}{3} \frac{\overline{d t}}{d \lambda}\left(\frac{\overline{\partial u}}{\partial y}+\frac{\overline{\partial v}}{\partial x}\right) \varepsilon\right]\left(n_{y}^{2}-n_{x}^{2}\right)-} \\
{\left[-\bar{p}+\left(\frac{2}{3}+\frac{2}{3} \frac{\overline{d t}}{d \lambda} \frac{\overline{\partial v}}{\partial y}\right) \varepsilon\right] n_{x} n_{y}= \pm \frac{\mu}{\sqrt{3}} \varepsilon} \\
\bar{v}_{n}=-\frac{\partial \bar{G} / \partial \bar{t}}{|\operatorname{grad} \bar{G}|}
\end{gathered}
$$

The system of differential equations (2.1)"-(2.3)" together with the boundary conditions (2.4)" - (2.7)" constitutes the general boundary-value problem of the spreading of a "viscous fluid".

In the main approximation $(\varepsilon=0)$, the original equations are simplified by decreasing the order of the system:

$$
\begin{gathered}
\frac{\partial \bar{p}}{\partial \bar{x}}=-\frac{2}{\sqrt{3}} \frac{1}{h} \frac{\bar{u}}{\sqrt{\bar{u}^{2}+\bar{v}^{2}}}, \\
\frac{\partial \bar{p}}{\partial \bar{y}}=-\frac{2}{\sqrt{3}} \frac{1}{h} \frac{\bar{v}}{\sqrt{\bar{u}^{2}+\bar{v}^{2}}}, \\
\frac{\partial \bar{u}}{\partial \bar{x}}+\frac{\partial \bar{v}}{\partial \bar{y}}-\frac{\partial \lambda}{\partial t}=0 .
\end{gathered}
$$

It is clear that the number of boundary conditions for the simplified problem of the "ideal fluid" also decreases.

Solution of the contact problem of plate tension (in a simplified formulation). Let's consider the problem of plastic stretching of a strip by forces applied on its "clamped" ends (Fig.1). In this case, different modes are possible, which are determined by the values of both the total compression force of the ends and the total tensile force.

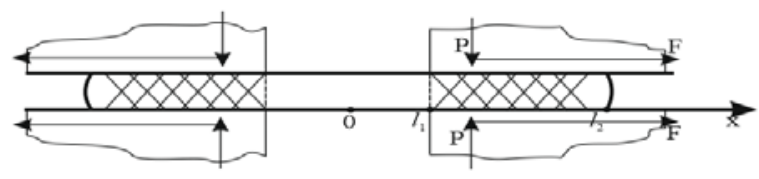

Fig.1. On the problem of complex plate loading.

\section{Solution}

Due to the symmetry of initial conditions let us formulate the boundary value problem for the area $0<x<l_{2}(t)$ : in the first part $0<x<l_{1}(t)$ the strip material is in a state of tension, and in another part $l_{1}(t)<x<l_{2}(t)$ the material subjected to complex loading in the form of pressing with stretching (or compression, depending on the direction of the applied force $F(t)$. Let's write out the differential equations of the boundary value problem in the domain $\left(l_{1}, l_{2}\right)$ :

$$
\frac{\partial u}{\partial x}-\frac{d \lambda}{d t}=0, \quad \frac{\partial p}{\partial x}=-\frac{2 \tau_{s}}{h} \frac{u-u_{0}}{\left|u-u_{0}\right|},
$$

where $u=u_{0}(t)$ - the unknown speed of movement of tools.

The boundary condition on the right free end:

$$
x=l_{2}(t): \quad p\left(l_{2}, t\right)=\sigma_{s},
$$

Integrating the equations (3.1), we get:

$$
\begin{gathered}
u(x, t)=-\frac{1}{h} \frac{d h}{d t}\left(x-x_{0}\right)+u_{0}(t), \\
p(x, t)= \begin{cases}-\frac{2 \tau_{s} x}{h}+c_{2}, & x \in\left(x_{0}, l_{2}(t)\right), \\
\frac{2 \tau_{s} x}{h}+c_{1}, & x \in\left(l_{1}(t), x_{0}\right)\end{cases}
\end{gathered}
$$

where $x=x_{0}(t)$ - the unknown branch line of the plastic flow in the band relative to the tool:

$$
x=x_{0}(t): u\left(x_{0}, t\right)=u_{0}(t) .
$$

To determine the three unknown values, two other conditions must be specified, in addition to the condition (3.2):

- the condition of continuity of the contact pressure at the point of branching of the flow;

- the some unknown, generally speaking, condition at the left end at $x=l_{1}(t)$.

From (3.2) we get:

$$
-\frac{2 \tau_{s}}{h} l_{2}+c_{2}=\sigma_{s}, c_{2}=\sigma_{s}+\frac{2 \tau_{s}}{h} l_{2} .
$$


From the condition of continuity of the contact pressure at the branch point of the flow $x=x_{0}(t)$, taking into account the condition (3.5), we find:

$$
c_{1}=c_{2}-\frac{2 \tau_{s}}{h}\left(2 x_{0}\right)=\sigma_{s}+\frac{2 \tau_{s}}{h}\left(l_{2}-2 x_{0}\right)
$$

Thus, we found a solution with an accuracy of up to $x_{0}(t)$. Let's look at a few cases.

A) Let the following condition be met

$$
u\left(l_{1}, t\right)=-\frac{1}{h} \frac{d h}{d t}\left(l_{1}-x_{0}\right)+u_{0}(t)=0,
$$

meaning that the flow rate of the layer material is zero on the left (stationary!) end of the contact area (i.e., the left end of the contact area moves at a speed $u_{0}(t)$, but there is no band flow rate at the point $x=l_{1}(t)$. In other words, there is a plastic pressing of the material in the capture area, and the Central part of the strip remains in its natural state (not deformed). It is clear that in this case we have a problem on the plastic sediment of the end part $\left(l_{1}(t) ; l_{2}(t)\right)$, which corresponds to the boundary condition of the left free end:

$$
x=l_{1}(t): \quad p(x, t)=\sigma_{s}
$$

from which we get that

$$
c_{1}=-\frac{2 \tau_{s}}{h} l_{1}+\sigma_{s}
$$

The condition (3.7) relates the rate of pressing ( $d h / d t$ ) and the rate of translational movement of the tool ( $u_{0}(t)$ ). In particular, it follows from (3.7) that if $u_{0}(t)=0$ we obtain that $x_{0} \equiv l_{1}$ (i.e., the left end of the part of the strip being deposited remains stationary). Comparing (3.6) and (3.9), we get:

$$
x_{0}(t)=\frac{l_{1}(t)+l_{2}(t)}{2}
$$

Now let's return to the condition (3.7), from which, taking into account the last ratio, we find such a value of the tool speed (in our case, the same as the speed of expansion of the middle part of the strip), at which the left end of the contact area is free of stresses:

$$
u_{0}(t)=\left(-\frac{1}{h} \frac{d h}{d t}\right) \frac{l_{2}-l_{1}}{2},
$$

or taking into account the incompressibility condition written in integral form

$$
\left(l_{1}-l_{2}\right) h=\left(l_{10}-l_{20}\right) h_{0},
$$

we get:

$$
u_{0}(t)=-\frac{d h}{d t} \frac{l_{20}-l_{10}}{2} h_{0}
$$

We need to find the laws of change $l_{1}(t)$ и $l_{2}(t)$ :

$$
\frac{d l_{1}}{d t}=u_{0}(t), \quad l_{1}\left(t_{0}\right)=l_{10} \Rightarrow l_{1}(t)=\int_{0}^{t} u_{0}(t) d t+l_{10}
$$

$\left.\left.\frac{d l_{2}}{d t}=u\left(l_{2}, t\right)=-\frac{1}{h} \frac{d h}{d t}\left(l_{2}-\frac{l_{2}+l_{1}}{2}\right)+u_{0}\right) t\right), l_{2}\left(t_{0}\right)=l_{20}$.

From the last system we get:

$$
\begin{gathered}
\frac{d\left(l_{2}-l_{1}\right)}{d t}=-\frac{1}{h} \frac{d h}{d t} \frac{1}{2}\left(l_{2}-l_{1}\right) \Rightarrow l_{2}-l_{1} \frac{\bar{c}_{1}}{\sqrt{h}} \\
\Rightarrow l_{2}=l_{1}+\frac{\bar{c}_{1}}{\sqrt{h}} .
\end{gathered}
$$

If we take into account the conditions (3.10), we get

$$
\begin{gathered}
\frac{d l_{1}}{d t}=u_{0}(t)=\left(-\frac{1}{h} \frac{d h}{d t}\right) \frac{1}{2}\left(l_{2}-l_{1}\right)=\left(-\frac{1}{h} \frac{d h}{d t}\right) \frac{1}{2} \frac{\bar{c}_{1}}{\sqrt{h}}, \\
l_{1}(t)=\frac{\bar{c}_{1}}{\sqrt{h}}+\bar{c}_{2} ; \quad l_{2}(t)=\frac{2 \bar{c}_{1}}{\sqrt{h}}+\bar{c}_{2} .
\end{gathered}
$$

Now let's substitute the initial data:

$$
\begin{gathered}
t=t_{0}: l_{10}=\frac{\bar{c}_{1}}{\sqrt{h_{0}}}+\bar{c}_{2} ; l_{20}=\frac{2 \bar{c}_{1}}{\sqrt{h_{0}}}+\bar{c}_{2} \Rightarrow \\
\bar{c}_{1}=\left(l_{20}-l_{10}\right) \sqrt{h_{0}} ; \bar{c}_{2}=2 l_{10}-l_{20} .
\end{gathered}
$$

The solution (3.12) takes the final form:

$$
\left\{\begin{array}{l}
l_{1}(t)=\left(l_{20}-l_{10}\right) \sqrt{\frac{h_{0}}{h}}+\left(2 l_{10}-l_{20}\right) \\
l_{2}(t)=2\left(l_{20}-l_{10}\right) \sqrt{\frac{h_{0}}{h}}+\left(2 l_{10}-l_{20}\right)
\end{array} .\right.
$$

B) Let the condition now be met

$$
u\left(l_{1}, t\right)=-\frac{1}{h} \frac{d h}{d t}\left(l_{1}-x_{0}\right)+u_{0}(t)>0
$$

that is, the left end of the (plastic) layer material in the contact area moves to the right, and $q\left(l_{1}\right)=q_{0}=-\sigma_{s}$. This condition necessarily leads to plastic stretching of the middle part of the strip $\left(0<x<l_{1}(t)\right)$, and, according to the condition of full plasticity [1]

$$
\sigma_{y y}=-p ; \sigma_{x x}=-p+\sigma_{s},
$$

we have:

$$
p\left(l_{1}, t\right)=0,
$$


thus, we got the missing boundary condition of the problem.

Rewrite (3.15) with (3.4), (3.6):

$$
\begin{aligned}
\frac{2 \tau_{s}}{h} l_{1}+c_{1} & =0 \Rightarrow \frac{2 \tau_{s}}{h} l_{1}+\left[\sigma_{s}+\frac{2 \tau_{s}}{h}\left(l_{2}-2 x_{0}\right)\right]=0 \\
x_{0}(t) & =\frac{\sigma_{s}}{\left(4 \tau_{s} / h\right)}+\frac{l_{1}+l_{2}}{2}>\frac{l_{1}+l_{2}}{2}
\end{aligned}
$$

that is, the branching line moves slightly towards the free-flowing end $x=l_{2}(t)$, and we can accept it with an accuracy up to infinitesimal (the theory of flow in a thin plastic layer [1] is characterized by an accuracy of order $\delta=h / l<<1)$ that:

$$
x_{0}=\frac{l_{1}+l_{2}}{2}
$$

If we substitute (3.16)' in (3.14), we get a condition for $u_{0}(t)$, when performing which simultaneously with the plastic pressing of the "clamped" end parts, the plastic stretching of the central part of the strip occurs:

$$
u_{0}(t)>-\frac{1}{h} \frac{d h}{d t}\left(x_{0}-l_{1}\right)=\left(-\frac{1}{h} \frac{d h}{d t}\right)\left(\frac{l_{2}-l_{1}}{2}\right)
$$

\section{Results}

Differential equation of the plastic layer free spreading on a plane and its particular solutions. Let us consider the process of free spreading of a thin plastic layer enclosed between two approaching surfaces of tool bodies. Such flows can be identified in a wide range of technological processes of material treatment by pressure, such as stamping and pressing of thin-walled structural elements, sheet rolling and others. In [1] using a two-dimensional averaging over the layer thickness, an efficient mathematical model to describe the flow process of a thin plastic layer is proposed.

Below, we will focus on the study of the kinematics of plastic layer spreading on a plane (which as a rule constitutes the main difficulty in application of boundary value problem), present a mathematical model of its description, and write out some particular solutions to this problem in analytical form.

It is known $[3,4]$ that the quasilinear parabolic equation

$$
\frac{\partial w}{\partial \tau}=\frac{\partial}{\partial x}\left(K(w) \frac{\partial w}{\partial x}\right)
$$

allows to describe fairly well various physical processes such as diffusion of heat in a continuous medium, heat and mass transfer, combustion and filtration. In particular, when in (4.1)

$$
K(w)=c w^{m},
$$

the equation is often used to describe the non-stationary heat transfer in a stationary medium, provided that the coefficient of thermal diffusivity is a power function of temperature. There are many exact solutions of equation (4.1), provided (4.2). For the cases, when $m=1,-1,-2,-4 / 3,-2 / 3$ such solutions can be found in [4]. Note, that the problem of the plastic layer free spreading on a plane is reduced to the study of the equation (4.1), in which $K(w)=\frac{1}{2} w^{2}$.

Let suppose that a thin layer of plastic material at the initial moment of time occupies a plane domain bounded by a piecewise smooth convex contour $y=\varphi\left(x, t_{0}\right) \equiv \varphi(x)$, symmetrical with respect to the horizontal axis $x$. Assume that the layer shrinks by the mean of two parallel rough planes that are converging along the common normal. In [2, 3] the following evolution equation for finding the contour $F(x, y, t)=y-\varphi(x, t)=0$ of the domain occupied by spreading plastic layer is obtained:

$$
\frac{\partial \varphi}{\partial t_{1}}-\varphi\left[1+\left(\frac{\partial \varphi}{\partial x}\right)^{2}\right]-\frac{\varphi^{2}}{2} \frac{\partial^{2} \varphi}{\partial x^{2}}=0,
$$

where $t_{1}(t)=\ln \left(h\left(t_{0}\right) / h(t)\right)$ is a dimensionless modified time $\left(\frac{d t_{1}}{d t}=-\frac{1}{h} \frac{d h}{d t}>0\right)$, and $h=h(t)$ is a well-known variation law of the layer thickness. In [3] the equation (4.3) is reduced to some particular form of nonlinear equation of heat conduction (4.1), with respect to the function $w(x, \tau), \quad\left(K(w) \equiv \frac{1}{2} w^{2}\right)$ :

$$
\begin{gathered}
\frac{\partial w}{\partial \tau}=\frac{1}{2} \frac{\partial}{\partial x}\left(w^{2} \frac{\partial w}{\partial x}\right), \varphi\left(x, \tau_{1}\right) \equiv e^{t_{1}} w(x, \tau), \tau \\
\equiv e^{2 t_{1}} / 2
\end{gathered}
$$

Let us consider another type of the evolution equation of spreading obtained from (4) using the substitution $q=w^{2}$ :

$$
4 \frac{\partial q}{\partial \tau}=2 q \frac{\partial^{2} q}{\partial x^{2}}+\left(\frac{\partial q}{\partial x}\right)^{2}
$$

In this paper all previously known exact solutions of the nonlinear equation (4.4) have been presented, namely:

1. $(x, \tau)=f(\tau) g(x)$, with:

1a) $f(\tau)=C_{1}, g(x)=C_{2}$ - the solution of the problem on the spreading of a strip parallel to the axis $x$;

1b) $f(\tau)=c_{1}, g=\sqrt[3]{c_{3} x+c_{4}}$ - the solution of the problem on spreading of the domain bounded by cubic parabola; 
1c) $2 f^{2}\left(\alpha_{1}+c_{0} \tau\right)=-1, \int \frac{g^{2} d g}{\sqrt{c_{0} g^{4}+\alpha_{2}}}= \pm x+\alpha_{3}$ -

the solution of the problem on spreading of the domain bounded by curve in the form of an elliptic integral;

2. $4(\tau-\gamma x)=C_{2}+(\gamma w)^{2}-2 C_{1} \gamma w+C_{1}^{2} \ln \left(C_{1}+\gamma w\right)^{2}$

- the solution of the problem on the spreading of the domain bounded by the parabola;

3. $q \equiv w^{2}=-\frac{x^{2}}{2\left(\tau+\tau_{0}\right)}+\frac{A_{3}}{\sqrt{\tau+\tau_{0}}}-$ the solution of the problem on the spreading of a plastic layer in the domain bounded by the ellipse whose major semi-axis lies on the $x$-axis;

4. $q \equiv w^{2}=-\frac{x^{2}}{2\left(\tau+\tau_{0}\right)}+\frac{A_{3} x^{2 / 3}}{\left(\tau+\tau_{0}\right)^{7 / 9}}$ - the generalized solution of the problem on the spreading of a plastic layer in the domain bounded by the ellipse;

5. $w(x, \tau)= \pm \sqrt{q^{2}}= \pm \frac{x}{\sqrt{2\left(\tau_{0}-\tau\right)}}$ - the solution of the problem on the spreading of a plastic layer in the domain in the form of a wedge;

6. $q \equiv w^{2}=\frac{x^{2}}{2\left(\tau_{0}-\tau\right)}+\frac{A_{3}}{\sqrt{\tau_{0}-\tau}} \quad$ - the solution of the problem on the spreading of the domain bounded by the hyperbola, whose real or imaginary axis, depending on the sign of $A_{3}$, is coincided with the axis $x$;

7. $q \equiv w^{2}=\frac{x^{2}}{2\left(\tau_{0}-\tau\right)}+\frac{A_{3} x^{2 / 3}}{\left(\tau_{0}-\tau\right)^{7 / 9}}$

the generalized solution of the problem on the spreading of a plastic layer bounded by a hyperbola.

\section{Conclusion}

The paper focuses on the theory of flow in a thin plastic layer, which was developed by A. A. Ilyushin and served as a guide in the calculations of technological processes of material treatment by pressure, such as stamping and pressing of thin-walled structural elements, thin-sheet rolling, and others. This paper reveals the current state of development of the mathematical modeling of contact problems of plastic flow in a thin layer.

The paper presents a solution to the contact problem of plastic stretching of the strip by forces applied on the "clamped" ends of the strip. The possible modes of the process were studied, which are determined by both the total compression force of the ends and the total tensile force.

The mathematical analogy between the process of the free spreading of a plastic layer on the plane and the process of heat transfer has been investigated. The exact particular solutions of the considered problem were obtained. The obtained results may be of interest for researchers in the field of nonlinear problems of heat and mass transfer as well as for engineers in the field of designing and carrying out calculation of processes of metals treatment by pressure.

The obtained results have interesting practical applications. In particular, analytical solutions of various generalizations of the classical L. Prandtl problem given in section 3 are of interest in the technique of studying the problem of straightening plates using uniaxial stretching beyond the elastic limit. It should be noted that straightening by stretching allows to obtain a high degree of flatness of thin wide strips and sheets made of high-strength steels and special alloys [6], which straightening by other methods does not give satisfactory results. The results may be used to describe a wide class of processes such as heat and-mass exchange in the technologies, biology and medicine, which are modeled using nonlinear parabolic equations, and for which there exist a mathematical analogy with the problems of spreading plastic layers considered in section 4 .

This work was carried out using equipment provided by the Center of Collective Use of MSUT "STANKIN".

\section{References}

1. A. Ilyushin, Proceedings (1946-1966).V.2. Plasticity (M.:Fizmatlit. 2004)

2. I.A. Kiyko, Theory of plastic flow (M.: MSU. 1978)

3. N. Belov, V. Kadymov, Mechanics of solids, 46(1), 36-46 (2011)

4. A. Polyanin, V. Zaitsev, Nonlinear equations of mathematical physics. Exact Solutions (Moscow. Fizmatlit, 2002)

5. V. Kadymov, Journ."Mathematical Methods in the Applied Sciences”, 38(16), 3421-3431 (2015) https://doi.org/10.1002/mma.3403

6. V. Kadymov, E. Yanovskaya, EPJ Web Conf., 224 (2019)

https://doi.org/10.1051/epjconf/201922401002 